\title{
DEGREE DISTANCE AND MINIMUM DEGREE
}

\author{
S. MUKWEMBI ${ }^{凶}$ and S. MUNYIRA
}

(Received 9 March 2012; accepted 11 April 2012; first published online 9 July 2012)

\begin{abstract}
Let $G$ be a finite connected graph of order $n$, minimum degree $\delta$ and diameter $d$. The degree distance $D^{\prime}(G)$ of $G$ is defined as $\sum_{\{u, v\} \subseteq V(G)}(\operatorname{deg} u+\operatorname{deg} v) d(u, v)$, where $\operatorname{deg} w$ is the degree of vertex $w$ and $d(u, v)$ denotes the distance between $u$ and $v$. In this paper, we find an asymptotically sharp upper bound on the degree distance in terms of order, minimum degree and diameter. In particular, we prove that

$$
D^{\prime}(G) \leq \frac{1}{4} d n\left(n-\frac{d}{3}(\delta+1)\right)^{2}+O\left(n^{3}\right) .
$$

As a corollary, we obtain the bound $D^{\prime}(G) \leq n^{4} /(9(\delta+1))+O\left(n^{3}\right)$ for a graph $G$ of order $n$ and minimum degree $\delta$. This result, apart from improving on a result of Dankelmann et al. ['On the degree distance of a graph', Discrete Appl. Math. 157 (2009), 2773-2777] for graphs of given order and minimum degree, completely settles a conjecture of Tomescu ['Some extremal properties of the degree distance of a graph', Discrete Appl. Math. 98 (1999), 159-163].
\end{abstract}

2010 Mathematics subject classification: primary 05C12.

Keywords and phrases: degree distance, minimum degree, diameter.

\section{Introduction}

Let $G$ be a connected graph with vertex set $V$ and edge set $E$. The degree distance $D^{\prime}(G)$ of $G$ is defined as

$$
\sum_{\{u, v\} \subseteq V}(\operatorname{deg} u+\operatorname{deg} v) d(u, v),
$$

where $\operatorname{deg} w$ is the degree of vertex $w$ and $d(u, v)$ denotes the distance between $u$ and $v$ in $G$.

The degree distance, a Schultz-type molecular topological index and a variant of the well-known and much studied Wiener index, seems to have been considered first by Dobrynin and Kochetova [5] in 1994 and practically at the same time by

This material is based upon work supported financially by the National Research Foundation. This work will be part of the second author's $\mathrm{PhD}$ thesis.

(C) 2012 Australian Mathematical Publishing Association Inc. 0004-9727/2012 \$16.00 
Gutman [6], who introduced it as a kind of a vertex-valency-weighted sum of the distances between all pairs of vertices in a graph. Gutman revealed that in the case of acyclic structures, the index is closely related to the Wiener index and reflects precisely the same structural features of a molecular graph as the Wiener index does.

Before 1994, the degree distance was encountered in connection with certain chemical applications [11, 12]. After 1994, the degree distance was investigated by several authors, for instance, Bucicovschi and Cioabă [1], Dankelmann et al. [3], Ilić et al. [8], Tomescu [13], and Tomescu [14]. In this paper we are concerned with upper bounds on the degree distance. Sharp upper and lower bounds on the degree distance for trees of given order have been completely determined (see, for example the recent survey in [4]). For general graphs, Dobrynin and Kochetova [5] conjectured that the largest degree distance of all connected graphs of order $n$ equals $n^{4} / 32+O\left(n^{3}\right)$. This was refuted by Tomescu [13], who showed that graphs consisting of two cliques of order approximately $n / 3$ joined by a path on approximately $n / 3$ vertices, have degree distance $n^{4} / 27+O\left(n^{3}\right)$. He then made the following attractive conjecture.

Conjecture 1.1 [13]. Let $G$ be a connected graph of order $n$. Then

$$
D^{\prime}(G) \leq \frac{n^{4}}{27}+O\left(n^{3}\right)
$$

Nine years after the announcement of this conjecture, Bucicovschi and Cioabă [1] commented that Tomescu's conjecture 'seems difficult at present time'. In the following year, Dankelmann et al. [3] considered this problem, and though they came close to proving the conjecture, their proof was unable to deal with the $O\left(n^{3}\right)$ error term. They proved the following bound.

THEOREM 1.2 [3]. If $G$ is a connected graph of order $n$, then

$$
D^{\prime}(G) \leq \frac{n^{4}}{27}+O\left(n^{7 / 2}\right) .
$$

Recently the method developed in [3] was improved by M. J. Morgan, S. Mukwembi and H. C. Swart ('On a conjecture by Tomescu', submitted for publication), leading to a complete solution of Tomescu's conjecture.

Since the degree distance can be considered as a weighted version of the Wiener index, comparisons between the two indices are inevitable. For trees of given order, these two parameters actually determine each other: Klein et al. [9] and Gutman [6] showed that, for every tree $T$ of order $n, D^{\prime}(T)=4 W(T)-n(n-1)$, where $W(G)=$ $\sum_{\{u, v\} \subseteq V} d(u, v)$ is the Wiener index of $G$. Since its introduction in the late 1940s by the chemist Harold Wiener in an attempt to analyse the chemical properties of paraffins (alkanes) [15], the mathematical properties of the Wiener index have been studied by several authors. One of the oldest results on upper bounds of this quantity is that amongst all connected graphs of given order, the path has the maximum Wiener index. For graphs of given minimum degree, this result was improved independently 
by several authors, among them Kouider and Winkler [10] and Dankelmann and Entringer [2], who proved the following bound.

Theorem $1.3[2,10]$. Let $G$ be a graph of order $n$ and minimum degree $\delta$. Then

$$
W(G) \leq \frac{n^{3}}{2(\delta+1)}+O\left(n^{2}\right),
$$

and this bound is best possible.

In light of Theorem 1.3 and the fact that the degree distance can be considered as a weighted version of the Wiener index, it is natural to look for a best upper bound on the degree distance of a connected graph of given order and minimum degree. To date, no work answering this question has been reported. The purpose of this paper is to contribute towards filling this gap. Our method is an improvement of the method initiated in [3]. We obtain an asymptotically sharp upper bound on the degree distance of a graph of given order and minimum degree. Our result, apart from being a strengthening of Theorem 1.2 and a theorem by Morgan et al. (see above), confirms and improves on Tomescu's conjecture (Conjecture 1.1) if minimum degree is prescribed.

The notation that we use is as follows. For a vertex $v$ of $G$, we denote by $D(v)$ the total distance or the status of $v$. That is, $D(v)=\sum_{x \in V(G)} d(v, x)$. The quantity $\operatorname{deg} v D(v)$ is denoted by $D^{\prime}(v)$. We denote the open neighbourhood of $v$ by $N(v)$, that is, $N(v)=\{x \in V(G) \mid d(x, v)=1\}$. The closed neigbourhood of $v$ in $G$, that is, $N(v) \cup\{v\}$, is denoted by $N[v]$. Here and in the following, we assume that the minimum degree $\delta$ is fixed.

The useful equation

$$
D^{\prime}(G)=\sum_{v \in V} D^{\prime}(v)
$$

was first observed by Tomescu [13].

\section{Results}

We begin by presenting a very simple but handy observation.

FACT 2.1. Let $G$ be a connected graph of order $n$, diameter $d$ and minimum degree $\delta$. If $v \in V(G)$, then $d \leq(3 /(\delta+1))(n-\operatorname{deg} v)+6$.

Proof. Assume that $v \in V(G)$ and let $P=v_{0}, v_{1}, \ldots, v_{d}$ be a diametric path of $G$. Let

$$
S:=\left\{v_{3 i+1} \mid i=0,1, \ldots,\left\lfloor\frac{d-1}{3}\right\rfloor\right\} .
$$

For each $x \in S$, choose any $\delta$ neighbours $x_{1}, x_{2}, \ldots, x_{\delta}$ of $x$ and denote the set $\left\{x, x_{1}, x_{2}, \ldots, x_{\delta}\right\}$ by $M[x]$. Let $M=\bigcup_{x \in S} M[x]$. Then

$$
|M|=(\delta+1)\left(\left\lfloor\frac{d-1}{3}\right\rfloor+1\right) \text {. }
$$


Note that by construction of $S, N[v] \cap M$ has at most $2 \delta+1$ vertices. Hence

$$
\begin{aligned}
n & \geq|M|+|N[v]|-|M \cap N[v]| \\
& \left.\geq(\delta+1)\left(\mid \frac{d-1}{3}\right\rfloor+1\right)+\operatorname{deg} v+1-(2 \delta+1) \\
& \geq(\delta+1) \frac{d}{3}+\operatorname{deg} v-2 \delta,
\end{aligned}
$$

and rearranging the terms completes the proof.

We will often use the following simple and straightforward result or a variation on it.

Proposition 2.2. Let $G$ be a connected graph of order $n$, diameter $d$ and fixed minimum degree $\delta$. If $v \in V(G)$, then

$$
D(v) \leq d\left(n-\frac{d}{6}(\delta+1)-\operatorname{deg} v\right)+O(n) .
$$

Proof. Denote the eccentricity of $v$ by $e$. For all $i=1,2, \ldots, e$, let $N_{i}:=\{x \in V(G) \mid$ $d(v, x)=i\}$ and $\left|N_{i}\right|=k_{i}$. Note that if $x \in N_{i}, i=2,3, \ldots, e-1$, then $N[x] \subseteq N_{i-1} \cup$ $N_{i} \cup N_{i+1}$ so that

$$
k_{i-1}+k_{i}+k_{i+1} \geq \delta+1
$$

Clearly,

$$
D(v)=1 k_{1}+2 k_{2}+\cdots+e k_{e} .
$$

We look at three cases separately.

Case 1: $e \equiv 0(\bmod 3)$. Subject to

$$
k_{1}=\operatorname{deg} v, \quad k_{i} \geq 1 \quad \text { for } i=2,3, \ldots, e,
$$

and

$$
k_{2}+k_{3}+k_{4} \geq \delta+1, k_{5}+k_{6}+k_{7} \geq \delta+1, \ldots, k_{e-4}+k_{e-3}+k_{e-2} \geq \delta+1,
$$

(2.1) is maximised for

$$
\begin{gathered}
k_{1}=\operatorname{deg} v, \quad k_{2}=1=k_{3}, \quad k_{4}=\delta-1, \quad k_{5}=1=k_{6}, \\
k_{7}=\delta-1, \ldots, k_{e-4}=1=k_{e-3}, \quad k_{e-2}=\delta-1, \quad k_{e-1}=1
\end{gathered}
$$

and

$$
k_{e}=n-\operatorname{deg} v-\frac{1}{3}(e-3)(\delta+1)-1-1
$$

This gives

$$
\begin{aligned}
D(v) \leq \operatorname{deg} & v+2+3+4(\delta-1)+5+6+7(\delta-1) \\
& +\cdots+(e-4)+(e-3)+(e-2)(\delta-1)+(e-1) \\
& +e\left(n-\operatorname{deg} v-\frac{1}{3}(e-3)(\delta+1)-2\right) \\
= & e\left(n-\frac{e}{6}(\delta+1)-\operatorname{deg} v\right)+O(n) .
\end{aligned}
$$


If $e=d-c$, where $c \in\{0,1, \ldots, 5\}$, then

$$
\begin{aligned}
D(v) & \leq(d-c)\left(n-\frac{d-c}{6}(\delta+1)-\operatorname{deg} v\right)+O(n) \\
& =d\left(n-\frac{d}{6}(\delta+1)-\operatorname{deg} v\right)+O(n),
\end{aligned}
$$

as desired. So assume that $e \leq d-6$. The function

$$
f(e)=e\left(n-\frac{e}{6}(\delta+1)-\operatorname{deg} v\right)
$$

is increasing in $e$ for all $e \leq(3 /(\delta+1))(n-\operatorname{deg} v)$. Note from Fact 2.1 that $d-6 \leq$ $(3 /(\delta+1))(n-\operatorname{deg} v)$. Hence

$$
D(v) \leq f(d-6)=d\left(n-\frac{d}{6}(\delta+1)-\operatorname{deg} v\right)+O(n),
$$

and the proposition is proved for this case. This completes Case 1.

The other cases, $e \equiv 1(\bmod 3)$ and $e \equiv 2(\bmod 3)$, are treated similarly.

THEOREM 2.3. Let $G$ be a connected graph of order $n$, diameter $d$ and fixed minimum degree $\delta$. Then

$$
D^{\prime}(G) \leq \frac{1}{4} d n\left(n-\frac{d}{3}(\delta+1)\right)^{2}+O\left(n^{3}\right)
$$

Moreover, this inequality is asymptotically tight.

Proof. Let $P: v_{0}, v_{1}, \ldots, v_{d}$ be a diametric path of $G$ and let $S \subset V(P)$ be the set

$$
S:=\left\{v_{3 i+1}: i=0,1,2, \ldots,\left\lfloor\frac{d-1}{3}\right\rfloor\right\} .
$$

For each $v \in S$, choose any $\delta$ neighbours $u_{1}, u_{2}, \ldots, u_{\delta}$ of $v$ and denote the set $\left\{v, u_{1}, u_{2}, \ldots, u_{\delta}\right\}$ by $M[v]$. Let $M=\bigcup_{v \in S} M[v]$. Then

$$
|M|=(\delta+1)\left(\left\lfloor\frac{d-1}{3}\right\rfloor+1\right) .
$$

Claim 1.

$$
\sum_{u \in M} D^{\prime}(u) \leq O\left(n^{3}\right)
$$

Proof of Claim 1. Let $S_{1} \subset S$ be the set $S_{1}=\left\{v_{j} \in S: j \equiv 1(\bmod 6)\right\}$. Let $S_{2}=$ $S-S_{1}$. Then for each $u, v \in S_{1}, \quad u \neq v$, we have $M[u] \cap M[v]=\emptyset$ and the neighbourhoods of $M[u]$ and $M[v]$ are also disjoint. Write the elements of $S_{1}$ as $S_{1}=\left\{w_{1}, w_{2}, \ldots, w_{\left|S_{1}\right|}\right\}$. For each $w_{j} \in S_{1}$, let $M\left[w_{j}\right]=\left\{w_{j}, u_{1}^{j}, u_{2}^{j}, \ldots, u_{\delta}^{j}\right\}$, where $u_{1}^{j}, u_{2}^{j}, \ldots, u_{\delta}^{j}$ are neighbours of $w_{j}$. Then

$$
n \geq\left(\operatorname{deg} w_{1}+1\right)+\left(\operatorname{deg} w_{2}+1\right)+\cdots+\left(\operatorname{deg} w_{\left|S_{1}\right|}+1\right)
$$


and, for $t=1,2, \ldots, \delta$,

$$
n \geq\left(\operatorname{deg} u_{t}^{1}+1\right)+\left(\operatorname{deg} u_{t}^{2}+1\right)+\cdots+\left(\operatorname{deg} u_{t}^{\left|S_{1}\right|}+1\right) .
$$

Summing yields

$$
(\delta+1) n \geq \sum_{x \in M\left[S_{1}\right]} \operatorname{deg} x+(\delta+1)\left|S_{1}\right|,
$$

where $M\left[S_{1}\right]=\sum_{u \in S_{1}} M[u]$.

Similarly,

$$
(\delta+1) n \geq \sum_{x \in M\left[S_{2}\right]} \operatorname{deg} x+(\delta+1)\left|S_{2}\right| .
$$

Thus

$$
\begin{aligned}
2(\delta+1) n & \geq \sum_{x \in M\left[S_{1}\right]} \operatorname{deg} x+\sum_{x \in M\left[S_{2}\right]} \operatorname{deg} x+(\delta+1)|S| \\
& =\sum_{x \in M\left[S_{1}\right]} \operatorname{deg} x+\sum_{x \in M\left[S_{2}\right]} \operatorname{deg} x+(\delta+1)\left(\left[\frac{d-1}{3}\right\rfloor+1\right) .
\end{aligned}
$$

Hence

$$
\begin{aligned}
\sum_{x \in M\left[S_{1}\right]} \operatorname{deg} x+\sum_{x \in M\left[S_{2}\right]} \operatorname{deg} x & \leq 2(\delta+1) n-(\delta+1)\left(\left\lfloor\frac{d-1}{3}\right\rfloor+1\right) \\
& =(\delta+1)\left(2 n-\left\lfloor\frac{d-1}{3}\right\rfloor-1\right) .
\end{aligned}
$$

Now for $u \in V(G), D(u) \leq(n-1) d \leq(n-1)^{2}$. It follows that

$$
\begin{aligned}
\sum_{v \in M} D^{\prime}(v) & =\sum_{v \in M} \operatorname{deg} v D(v) \\
& =\sum_{v \in M\left[S_{1}\right]} \operatorname{deg} v D(v)+\sum_{v \in M\left[S_{2}\right]} \operatorname{deg} v D(v) \\
& \leq(n-1)^{2}\left(\sum_{v \in M\left[S_{1}\right]} \operatorname{deg} v+\sum_{v \in M\left[S_{2}\right]} \operatorname{deg} v\right) \\
& \leq(n-1)^{2}(\delta+1)\left(2 n-\left\lfloor\frac{d-1}{3}\right\rfloor-1\right) \\
& =O\left(n^{3}\right),
\end{aligned}
$$

as required and so Claim 1 is proved.

Let $C$ be a maximum set of disjoint pairs of vertices from $V-M$ which lie at a distance at least 3 , that is, if $\{a, b\} \in C$, then $d(a, b) \geq 3$. If $\{a, b\} \in C$ we say $a$ and $b$ are partners. Finally, let $K$ be the remaining vertices of $G$, that is, $K=V-M-\{x$ : $x \in\{a, b\} \in C\}$. Let $|K|=k$, and $|C|=c$. Then

$$
n=(\delta+1)\left(\left[\frac{d-1}{3}\right\rfloor+1\right)+2 c+k .
$$


FACT 2.4. Let $\{a, b\} \in C$. Then $\operatorname{deg} a+\operatorname{deg} b \leq n-(d / 3)(\delta+1)+O(1)$.

Proof of Fact 2.4. Note that $N[a] \cap N[b]=\emptyset$, since $d(a, b) \geq 3$. Also, each of the two vertices can be adjacent to at most $2 \delta+1$ vertices on $M$. Thus,

$$
\begin{aligned}
n & \geq \operatorname{deg} a+1+\operatorname{deg} b+1+|M|-2(2 \delta+1) \\
& =\operatorname{deg} a+\operatorname{deg} b+(\delta+1)\left(\left\lfloor\frac{d-1}{3}\right\rfloor+1\right)-4 \delta,
\end{aligned}
$$

and rearranging the terms completes the proof of Fact 2.4.

Now consider two cases.

Case 1: $k \leq 1$. For $x \in K, D(x) \leq(n-1)^{2}$, so $D^{\prime}(x) \leq(n-1)^{3}$. Thus $\sum_{x \in K} D^{\prime}(x)=$ $O\left(n^{3}\right)$.

Claim 2. If $\{a, b\} \in C$, then

$$
D^{\prime}(a)+D^{\prime}(b) \leq \frac{1}{2} d n\left(n-\frac{d}{3}(\delta+1)\right)+O\left(n^{2}\right) .
$$

Proof of Claim 2. By Proposition 2.2,

$$
D(a) \leq d\left(n-\frac{d}{6}(\delta+1)-\operatorname{deg} a\right)+O(n) .
$$

It follows that

$$
D^{\prime}(a) \leq \operatorname{deg} a\left(d\left(n-\frac{d}{6}(\delta+1)-\operatorname{deg} a\right)\right)+O\left(n^{2}\right)
$$

Similarly,

$$
D^{\prime}(b) \leq \operatorname{deg} b\left(d\left(n-\frac{d}{6}(\delta+1)-\operatorname{deg} b\right)\right)+O\left(n^{2}\right) .
$$

Thus,

$$
\begin{aligned}
D^{\prime}(a)+D^{\prime}(b) \leq & \operatorname{deg} a\left(d\left(n-\frac{d}{6}(\delta+1)-\operatorname{deg} a\right)\right) \\
& \quad+\operatorname{deg} b\left(d\left(n-\frac{d}{6}(\delta+1)-\operatorname{deg} b\right)\right)+O\left(n^{2}\right) \\
= & d\left((\operatorname{deg} a+\operatorname{deg} b)\left(n-\frac{d}{6}(\delta+1)\right)-\left((\operatorname{deg} a)^{2}+(\operatorname{deg} b)^{2}\right)\right)+O\left(n^{2}\right) \\
\leq & d\left((\operatorname{deg} a+\operatorname{deg} b)\left(n-\frac{d}{6}(\delta+1)\right)-\frac{1}{2}(\operatorname{deg} a+\operatorname{deg} b)^{2}\right)+O\left(n^{2}\right) .
\end{aligned}
$$

Denote $\operatorname{deg} a+\operatorname{deg} b$ by $x$ and let

$$
f(x)=d\left(x\left(n-\frac{d}{6}(\delta+1)\right)-\frac{1}{2} x^{2}\right) .
$$


Then by Fact 2.4, $x \leq n-(d / 3)(\delta+1)+O(1)$. A simple differentiation shows that $f$ is increasing for all $x \leq n-(d / 6)(\delta+1)$. Hence $f$ attains its maximum for $x=$ $n-(d / 3)(\delta+1)+O(1)$, to give

$$
\begin{aligned}
D^{\prime}(a)+D^{\prime}(b) & \leq f\left(n-\frac{d}{3}(\delta+1)+O(1)\right) \\
& =\frac{1}{2} d n\left(n-\frac{d}{3}(\delta+1)\right)+O\left(n^{2}\right),
\end{aligned}
$$

and Claim 2 is proved.

From (2.2),

$$
c=\frac{1}{2}\left(n-(\delta+1)\left(\left[\frac{d-1}{3}\right\rfloor+1\right)-k\right) .
$$

Hence, since $k \leq 1$,

$$
c=\frac{1}{2}\left(n-\frac{d}{3}(\delta+1)\right)+O(1) .
$$

This, in conjunction with Claim 2, yields

$$
\begin{aligned}
\sum_{\{a, b\} \in C}\left(D^{\prime}(a)+D^{\prime}(b)\right) & \leq c\left(\frac{1}{2} d n\left(n-\frac{d}{3}(\delta+1)\right)+O\left(n^{2}\right)\right) \\
& =\left(\frac{1}{2}\left(n-\frac{d}{3}(\delta+1)\right)+O(1)\right)\left(\frac{1}{2} d n\left(n-\frac{d}{3}(\delta+1)\right)+O\left(n^{2}\right)\right) \\
& =\frac{1}{4} d n\left(n-\frac{d}{3}(\delta+1)\right)^{2}+O\left(n^{3}\right) .
\end{aligned}
$$

Hence

$$
\begin{aligned}
D^{\prime}(G) & =\sum_{\{a, b\} \in C}\left(D^{\prime}(a)+D^{\prime}(b)\right)+\sum_{x \in K} D^{\prime}(x)+\sum_{v \in M} D^{\prime}(v) \\
& \leq \frac{1}{4} d n\left(n-\frac{d}{3}(\delta+1)\right)^{2}+O\left(n^{3}\right)+O\left(n^{3}\right)+O\left(n^{3}\right),
\end{aligned}
$$

and so the theorem is proved for Case 1.

Case 2: $k \geq 2$. Now the pairs of vertices in $C$ will be partitioned further. Fix a vertex $x \in K$. For each pair $\{a, b\} \in C$, choose the vertex closer to $x$; if $d(a, x)=d(b, x)$ arbitrarily choose one of the vertices. Let $A$ be the set of all these vertices closer to $x$, and $B$ be the set of partners of these vertices in $A$, so $|A|=|B|=c$. Furthermore, let $A_{1}\left(B_{1}\right)$ be the set of vertices $w \in A(B)$ whose partner is at a distance at most 9 from $w$. Let $c_{1}=\left|A_{1}\right|=\left|B_{1}\right|$.

Claim 3. For all $u, v \in A \cup K, d(u, v) \leq 8$.

Proof of Claim 3. Since $C$ is a maximum set of pairs of vertices of distance at least 3 , any two vertices of $K$ must be at a distance of at most 2 . We show that $d(a, x) \leq 4$ for all $a \in A$. Suppose, to the contrary, that there exists a vertex $a \in A$ for which $d(a, x) \geq 5$. 
Let $b$ be the partner of $a$. By definition of $A, d(x, b) \geq 5$. Now consider another vertex $x^{\prime} \in K, x \neq x^{\prime}$. Since $d\left(x, x^{\prime}\right) \leq 2$,

$$
5 \leq d(b, x) \leq d\left(b, x^{\prime}\right)+d\left(x, x^{\prime}\right) \leq d\left(b, x^{\prime}\right)+2,
$$

which implies that $d\left(b, x^{\prime}\right) \geq 3$. This contradicts the maximality of $C$ since $\{a, b\}$ will be replaced by $\{a, x\}$ and $\left\{b, x^{\prime}\right\}$. Hence $d(a, x) \leq 4$, for each $a \in A$. Thus for $u, v \in A, d(u, v) \leq d(u, x)+d(x, v) \leq 8$.

Claim 4. For all $x \in K$,

$$
D^{\prime}(x) \leq d\left(n-\frac{d}{3}(\delta+1)-c\right)\left(n-c-c_{1}-k-\frac{d}{6}(\delta+1)\right)+O\left(n^{2}\right) .
$$

Proof of Claim 4. By Claim 3, all $c+k$ vertices in $A \cup K$ lie within a distance of 8 from each vertex $x \in K$. This implies that all the $c_{1}$ vertices in $B_{1}$ lie within a distance of $9+8$ from $x$. Thus, as in Proposition 2.2,

$$
\begin{gathered}
D(x) \leq 8(c+k)+17 c_{1}+18+19+20(\delta-1)+21+22+23(\delta-1) \\
+\cdots+d\left(n-c-k-c_{1}-\frac{d}{3}(\delta+1)\right) \\
=d\left(n-c-c_{1}-k-\frac{d}{6}(\delta+1)\right)+O(n) .
\end{gathered}
$$

In order to find a bound on the degree of $x$ we use a counting argument. Note that $x$ can have at most $2 \delta+1$ neighbours in $M$. By definition of $A$ and $B, x$ cannot be adjacent to two vertices, $w$ and $z$, where $w \in A$ is a partner of $z \in B$, since $d(w, z) \geq 3$. Thus, $x$ is adjacent to at most $c$ vertices in $A \cup B$. It follows that

$$
\begin{aligned}
n & \geq \operatorname{deg} x+|M|-(2 \delta+1)+|A \cup B|-c \\
& =\operatorname{deg} x+(\delta+1)\left(\left\lfloor\frac{d-1}{3}\right\rfloor+1\right)-(2 \delta+1)+c \\
& =\operatorname{deg} x+\frac{d}{3}(\delta+1)+c+O(1) .
\end{aligned}
$$

Hence deg $x \leq n-(d / 3)(\delta+1)-c+O(1)$. Therefore,

$$
\begin{aligned}
D^{\prime}(x) & =\operatorname{deg} x D(x) \\
& \leq d\left(n-\frac{d}{3}(\delta+1)-c\right)\left(n-c-c_{1}-k-\frac{d}{6}(\delta+1)\right)+O\left(n^{2}\right),
\end{aligned}
$$

and this proves Claim 4.

We now turn to finding an upper bound on the contribution of the pairs in $C$ to the degree distance. We abuse notation and write $\{a, b\} \in A_{1} \cup B_{1}$ if $a$ and $b$ are partners, that is, $\{a, b\} \in C$, with $a \in A_{1}$ and $b \in B_{1}$. Note that

$$
\sum_{\{a, b\} \in C}\left(D^{\prime}(a)+D^{\prime}(b)\right)=\sum_{\{a, b\} \in A_{1} \cup B_{1}}\left(D^{\prime}(a)+D^{\prime}(b)\right)+\sum_{\{a, b\} \in\left(A-A_{1}\right) \cup\left(B-B_{1}\right)}\left(D^{\prime}(a)+D^{\prime}(b)\right) .
$$

We first consider the set $A_{1} \cup B_{1}$. 
Claim 5. Let $\{a, b\} \in C$. If $d(a, b) \leq 9$, that is, if $\{a, b\} \in A_{1} \cup B_{1}$, then

$$
D^{\prime}(a)+D^{\prime}(b) \leq d\left(n-\frac{d}{3}(\delta+1)\right)\left(n-c-c_{1}-k-\frac{d}{6}(\delta+1)\right)+O\left(n^{2}\right) .
$$

Proof of Claim 5. We first show that any two vertices in $A \cup K \cup B_{1}$ lie within a distance of 26 from each other. By Claim 3, any two vertices in $A \cup K$ lie within a distance of 8 from each other. Now assume that $b, v \in B_{1}$, and let $a$ and $u$ be the partners of $b$ and $v$ in $A_{1}$, respectively. Then $d(b, v) \leq d(b, a)+d(a, u)+d(u, v) \leq$ $9+8+9=26$. Thus any two vertices in $B_{1}$ are within a distance of 26 from each other. Now let $a \in A \cup K$ and $b \in B_{1}$, and let $u$ be the partner of $b$ in $A_{1} \subseteq A$. Then $d(a, b) \leq d(a, u)+d(u, b) \leq 8+9<26$. Hence any two vertices in $A \cup K \cup B_{1}$ lie within a distance of 26 from each other.

Now let $w \in A_{1} \cup B_{1}$. Since $w$ is in $A \cup K \cup B_{1}$, all the $c+k+c_{1}-1$ vertices in $A \cup K \cup B_{1}$ lie within a distance of 26 from $w$. It follows, as in Proposition 2.2, that

$$
\begin{aligned}
D(w) \leq & 26\left(c+k+c_{1}-1\right)+27+28+29(\delta-1)+30+31+32(\delta-1)+\cdots \\
& +d\left(n-c-c_{1}-k-\frac{d}{3}(\delta+1)\right) \\
= & d\left(n-c-c_{1}-k-\frac{d}{6}(\delta+1)\right)+O(n) .
\end{aligned}
$$

Thus, if $\{a, b\}$ is a pair in $A_{1} \cup B_{1}$, then

$$
\begin{aligned}
D^{\prime}(a)+D^{\prime}(b) \leq & \operatorname{deg} a\left(d\left(n-c-c_{1}-k-\frac{d}{6}(\delta+1)\right)+O(n)\right) \\
& \quad+\operatorname{deg} b\left(d\left(n-c-c_{1}-k-\frac{d}{6}(\delta+1)\right)+O(n)\right) \\
= & (\operatorname{deg} a+\operatorname{deg} b)\left(d\left(n-c-c_{1}-k-\frac{d}{6}(\delta+1)\right)+O(n)\right) .
\end{aligned}
$$

By Fact 2.4, $\operatorname{deg} a+\operatorname{deg} b \leq n-(d / 3)(\delta+1)+O(1)$. Therefore,

$$
\begin{aligned}
D^{\prime}(a)+D^{\prime}(b) & \leq\left(n-\frac{d}{3}(\delta+1)+O(1)\right)\left(d\left(n-c-c_{1}-k-\frac{d}{6}(\delta+1)\right)+O(n)\right) \\
& =d\left(n-\frac{d}{3}(\delta+1)\right)\left(n-c-c_{1}-k-\frac{d}{6}(\delta+1)\right)+O\left(n^{2}\right),
\end{aligned}
$$

and Claim 5 is proved.

Now consider pairs $\{a, b\}$ of vertices in $C$ which are not in $A_{1} \cup B_{1}$.

Claim 6. Let $\{a, b\} \in C$. If $d(a, b) \geq 10$, that is, if $\{a, b\} \in\left(A-A_{1}\right) \cup\left(B-B_{1}\right)$, then

$$
\begin{array}{r}
D^{\prime}(a)+D^{\prime}(b) \leq d(c+k)\left(n-c-c_{1}-k-\frac{d}{6}(\delta+1)\right) \\
+c d\left(n-\frac{d}{6}(\delta+1)-c\right)+O\left(n^{2}\right) .
\end{array}
$$


Proof of Claim 6. We consider vertices from $A-A_{1}$ and from $B-B_{1}$ separately. Let $a \in A-A_{1}$. Then as in Claim 5, all the $c+k-1$ vertices in $A \cup K$ lie at a distance of 8 from $a$ and all the $c_{1}$ vertices in $B_{1}$ lie within a distance of $9+8=17$ from $a$. Thus, as in Proposition 2.2,

$$
\begin{aligned}
D(a) \leq & 8(c+k-1)+17 c_{1}+18+19+20(\delta-1)+21+22+23(\delta-1) \\
& +\cdots+d\left(n-c-c_{1}-k-\frac{d}{3}(\delta+1)\right) \\
= & d\left(n-c-c_{1}-k-\frac{d}{6}(\delta+1)\right)+O(n) .
\end{aligned}
$$

We now find a bound on the degree of $a$. By definition of $C, a$ cannot be adjacent to both $w$ and $u$, where $w \in A$ is a partner of $u \in B$, since $d(w, u) \geq 3$. Hence $a$ is adjacent to at most $c-1$ vertices in $A \cup B$. Further, $a$ is adjacent to at most $2 \delta+1$ vertices in $M$ and has at most $k$ neighbours in $K$. Thus,

$$
\operatorname{deg} a \leq c-1+2 \delta+1+k=c+2 \delta+k .
$$

It follows that

$$
\begin{aligned}
D^{\prime}(a) & =\operatorname{deg} a D(a) \\
& \leq(c+k+2 \delta)\left(d\left(n-c-c_{1}-k-\frac{d}{6}(\delta+1)\right)+O\left(n^{2}\right)\right) \\
& =d(c+k)\left(n-c-c_{1}-k-\frac{d}{6}(\delta+1)\right)+O\left(n^{2}\right) .
\end{aligned}
$$

Now let $b \in B-B_{1}$. By Proposition 2.2,

$$
D(b) \leq d\left(n-\frac{d}{6}(\delta+1)-\operatorname{deg} b\right)+O(n),
$$

and so

$$
D^{\prime}(b) \leq \operatorname{deg} b\left(d\left(n-\frac{d}{6}(\delta+1)-\operatorname{deg} b\right)\right)+O\left(n^{2}\right) .
$$

We first maximise

$$
\operatorname{deg} b\left(d\left(n-\frac{d}{6}(\delta+1)-\operatorname{deg} b\right)\right)
$$

with respect to $\operatorname{deg} b$. Let

$$
f(x):=x\left(d\left(n-\frac{d}{6}(\delta+1)-x\right)\right),
$$

where $x=\operatorname{deg} b$. A simple differentiation shows that $f$ is increasing for $x \leq$ $\frac{1}{2}(n-(d / 6)(\delta+1))$. We find an upper bound on $x$, that is, on $\operatorname{deg} b$. Note that as above, $b$ can be adjacent to at most $c-1$ vertices in $A \cup B$, and has at most $2 \delta+1$ neighbours in $M$. We show that $b$ cannot be adjacent to any vertex in $K$. Suppose to the contrary 
that $y \in K$ and $d(b, y)=1$. Recall that $a$ is the partner of $b$ and $d(a, b) \geq 10$. By Claim 3, $d(a, y) \leq 8$. Hence $10 \leq d(a, b) \leq d(b, y)+d(y, a) \leq 1+8$, a contradiction. Thus, $b$ cannot be adjacent to any vertex in $K$. We conclude that

$$
\operatorname{deg} b \leq c-1+2 \delta+1=c+2 \delta .
$$

We look at two cases separately. First assume that $\operatorname{deg} b=c+j$, where $j \in$ $\{1,2, \ldots, 2 \delta\}$. Then

$$
\begin{aligned}
f(\operatorname{deg} b) & =f(c+j) \\
& =(c+j)\left(d\left(n-\frac{d}{6}(\delta+1)-(c+j)\right)\right) \\
& =c d\left(n-\frac{d}{6}(\delta+1)-c\right)+O\left(n^{2}\right) .
\end{aligned}
$$

Second, assume that $\operatorname{deg} b \leq c$. From (2.2), and since $k \geq 2$ and $\lfloor(d-1) / 3\rfloor+1 \geq d / 3$,

$$
c=\frac{1}{2}\left(n-(\delta+1)\left(\left\lfloor\frac{d-1}{3}\right\rfloor+1\right)-k\right) \leq \frac{1}{2}\left(n-\frac{d}{3}(\delta+1)-2\right) .
$$

Notice that

$$
\frac{1}{2}\left(n-\frac{d}{3}(\delta+1)-2\right) \leq \frac{1}{2}\left(n-\frac{d}{6}(\delta+1)\right)
$$

and so $f$ is increasing in $[1, c]$. Therefore,

$$
f(\operatorname{deg} b) \leq f(c)=c d\left(n-\frac{d}{6}(\delta+1)-c\right),
$$

for this case. Comparing this with (2.5), we get that

$$
f(\operatorname{deg} b) \leq c d\left(n-\frac{d}{6}(\delta+1)-c\right)+O\left(n^{2}\right) .
$$

Thus, from (2.4),

$$
D^{\prime}(b) \leq c d\left(n-\frac{d}{6}(\delta+1)-c\right)+O\left(n^{2}\right)
$$

Combining this with (2.3),

$$
\begin{array}{r}
D^{\prime}(a)+D^{\prime}(b) \leq d(c+k)\left(n-c-c_{1}-k-\frac{d}{6}(\delta+1)\right) \\
+c d\left(n-\frac{d}{6}(\delta+1)-c\right)+O\left(n^{2}\right),
\end{array}
$$

and Claim 6 is proved. 
Using Claims 1 and 4-6 we bound $D^{\prime}(G)$ as follows. Note that

$$
\begin{aligned}
D^{\prime}(G)= & \sum_{u \in M} D^{\prime}(u)+\sum_{x \in K} D^{\prime}(x)+\sum_{\{a, b\} \in C}\left(D^{\prime}(a)+D^{\prime}(b)\right) \\
\leq & d k\left(n-\frac{d}{3}(\delta+1)-c\right)\left(n-c-c_{1}-k-\frac{d}{6}(\delta+1)\right) \\
& +c_{1}\left(d\left(n-\frac{d}{3}(\delta+1)\right)\left(n-c-c_{1}-k-\frac{d}{6}(\delta+1)\right)\right) \\
& +\left(c-c_{1}\right)\left(d(c+k)\left(n-c-c_{1}-k-\frac{d}{6}(\delta+1)\right)\right. \\
& \left.+c d\left(n-\frac{d}{6}(\delta+1)-c\right)\right)+O\left(n^{3}\right) \\
= & d k\left(n-\frac{d}{3}(\delta+1)-c\right)\left(n-c-c_{1}-k-\frac{d}{6}(\delta+1)\right) \\
& +c_{1}\left(d\left(n-\frac{d}{3}(\delta+1)\right)\left(n-c-c_{1}-k-\frac{d}{6}(\delta+1)\right)\right) \\
& +d\left(c-c_{1}\right)\left((c+k)\left(n-c-k-\frac{d}{6}(\delta+1)\right)\right. \\
& \left.\quad c_{1}(c+k)+c\left(n-\frac{d}{6}(\delta+1)-c\right)\right)+O\left(n^{3}\right) .
\end{aligned}
$$

For easy calculation in maximising this term, we note that $c-c_{1} \geq 0$, and that by (2.2), $n-c-k-(d / 6)(\delta+1) \geq 0$. Hence the last term in the previous inequalities,

$$
d\left(c-c_{1}\right)\left((c+k)\left(n-c-k-\frac{d}{6}(\delta+1)\right)-c_{1}(c+k)+c\left(n-\frac{d}{6}(\delta+1)-c\right)\right),
$$

is at most

$$
d\left(c-c_{1}\right)\left((c+k+1)\left(n-c-k-\frac{d}{6}(\delta+1)\right)-c_{1}(c+k)+c\left(n-\frac{d}{6}(\delta+1)-c\right)\right) .
$$

It follows that

$$
\begin{aligned}
D^{\prime}(G) \leq d k & \left(n-\frac{d}{3}(\delta+1)-c\right)\left(n-c-c_{1}-k-\frac{d}{6}(\delta+1)\right) \\
& +c_{1}\left(d\left(n-\frac{d}{3}(\delta+1)\right)\left(n-c-c_{1}-k-\frac{d}{6}(\delta+1)\right)\right) \\
& +d\left(c-c_{1}\right)\left((c+k+1)\left(n-c-k-\frac{d}{6}(\delta+1)\right)-c_{1}(c+k)\right. \\
& \left.+c\left(n-\frac{d}{6}(\delta+1)-c\right)\right)+O\left(n^{3}\right) .
\end{aligned}
$$


Let $g\left(n, d, c, c_{1}\right)$ be the function

$$
\begin{array}{rl}
g\left(n, d, c, c_{1}\right):=d & k\left(n-\frac{d}{3}(\delta+1)-c\right)\left(n-c-c_{1}-k-\frac{d}{6}(\delta+1)\right) \\
& +c_{1}\left(d\left(n-\frac{d}{3}(\delta+1)\right)\left(n-c-c_{1}-k-\frac{d}{6}(\delta+1)\right)\right) \\
& +d\left(c-c_{1}\right)\left((c+k+1)\left(n-c-k-\frac{d}{6}(\delta+1)\right)-c_{1}(c+k)\right. \\
& \left.+c\left(n-\frac{d}{6}(\delta+1)-c\right)\right) .
\end{array}
$$

We first maximise $g$ subject to $c_{1}$, keeping the other variables fixed. It is easy to verify, using (2.2), that the derivative

$$
\frac{d g}{d c_{1}}=-d k\left(n-\frac{d}{3}(\delta+1)\right)-d c\left(n-\frac{d}{3}(\delta+1)-2 c+c_{1}\right)-d\left(c+\frac{d}{6}(\delta+1)\right)
$$

is negative. Therefore, $g$ is decreasing in $c_{1}$. Thus, in conjunction with (2.2),

$$
\begin{aligned}
g\left(n, d, c, c_{1}\right) \leq & g(n, d, c, 0) \\
= & d k\left(n-\frac{d}{3}(\delta+1)-c\right)\left(n-c-k-\frac{d}{6}(\delta+1)\right) \\
& +d c\left((c+k+1)\left(n-c-k-\frac{d}{6}(\delta+1)\right)+c\left(n-\frac{d}{6}(\delta+1)-c\right)\right) \\
=d & \left(n-\frac{d}{3}(\delta+1)-2 c\right)\left(n-\frac{d}{3}(\delta+1)-c\right) \\
& \times\left(n-c-\left(n-\frac{d}{3}(\delta+1)-2 c\right)-\frac{d}{6}(\delta+1)\right) \\
& +d c\left(\left(c+\left(n-\frac{d}{3}(\delta+1)-2 c\right)+1\right)\right. \\
& \left.\times\left(n-c-\left(n-\frac{d}{3}(\delta+1)-2 c\right)-\frac{d}{6}(\delta+1)\right)\right) \\
& +d c\left(c\left(n-\frac{d}{6}(\delta+1)-c\right)\right)+O\left(n^{3}\right) \\
= & d\left(n-\frac{d}{3}(\delta+1)-2 c\right)\left(n-\frac{d}{3}(\delta+1)-c\right)\left(c+\frac{d}{6}(\delta+1)\right) \\
& +d c\left(\left(n-\frac{d}{3}(\delta+1)-c+1\right)\left(c+\frac{d}{6}(\delta+1)\right)\right) \\
& +d c\left(c\left(n-\frac{d}{6}(\delta+1)-c\right)\right)+O\left(n^{3}\right) \\
=d( & \left(n-\frac{d}{3}(\delta+1)-c\right)^{2}\left(c+\frac{d}{6}(\delta+1)\right) \\
& \left.+c^{2}\left(n-\frac{d}{6}(\delta+1)-c\right)\right)+O\left(n^{3}\right) .
\end{aligned}
$$


A simple differentiation with respect to $c$ shows that the function

$$
\left(n-\frac{d}{3}(\delta+1)-c\right)^{2}\left(c+\frac{d}{6}(\delta+1)\right)+c^{2}\left(n-\frac{d}{6}(\delta+1)-c\right)
$$

attains its maximum for $c=\frac{1}{2}(n-(d / 3)(\delta+1))$ to give

$$
\left(n-\frac{d}{3}(\delta+1)-c\right)^{2}\left(c+\frac{d}{6}(\delta+1)\right)+c^{2}\left(n-\frac{d}{6}(\delta+1)-c\right) \leq \frac{n}{4}\left(n-\frac{d}{3}(\delta+1)\right)^{2} .
$$

Hence

$$
g\left(n, d, c, c_{1}\right) \leq \frac{1}{4} d n\left(n-\frac{d}{3}(\delta+1)\right)^{2}+O\left(n^{3}\right)
$$

and so

$$
D^{\prime}(G) \leq g\left(n, d, c, c_{1}\right)+O\left(n^{3}\right) \leq \frac{1}{4} d n\left(n-\frac{d}{3}(\delta+1)\right)^{2}+O\left(n^{3}\right),
$$

and Case 2 of Theorem 2.3 is proved.

To see that the bound is asymptotically sharp, consider the graph $G_{n, d, \delta}, d \equiv 1$ (mod 3), constructed as follows. First, let $H$ be the graph with diameter $d-2$ obtained as follows: $V(H)=V_{0} \cup V_{1} \cup \cdots \cup V_{d-2}$, where

$$
\left|V_{i}\right|= \begin{cases}1 & \text { if } i \equiv 0 \text { or } 2(\bmod 3), \\ \delta-1 & \text { otherwise }\end{cases}
$$

and two distinct vertices $v \in V_{i}, v^{\prime} \in V_{j}$ are joined by an edge if and only if $|j-i| \leq 1$. Let the only vertex in $V_{0}$ be $v_{0}$ and the only vertex in $V_{d-2}$ be $v_{d-2}$. Now let $H_{1}$ be the complete graph on $\left\lceil\frac{1}{2}\left(n-\frac{1}{3}(d-1)(\delta+1)\right)\right\rceil$ vertices and $H_{2}$ the complete graph on $\left\lfloor\frac{1}{2}\left(n-\frac{1}{3}(d-1)(\delta+1)\right)\right\rfloor$ vertices. The graph $G_{n, d, \delta}$ is obtained by joining the vertex $v_{0}$ in $H$ to every vertex in $H_{1}$ and joining the vertex $v_{d-2}$ in $H$ to every vertex in $H_{2}$. Then $G_{n, d, \delta}$ has diameter $d$, minimum degree $\delta$ and degree distance at least $(1 / 4) \operatorname{dn}(n-(d / 3)(\delta+1))^{2}$, as desired.

Finally, the result below gives a strengthening of the bound in [3] and completely settles a conjecture of Tomescu [13].

Corollary 2.5. Let $G$ be a connected graph of order $n$ and minimum degree $\delta$. Then

$$
D^{\prime}(G) \leq \frac{n^{4}}{9(\delta+1)}+O\left(n^{3}\right)
$$

Moreover for a fixed $\delta$, this inequality is asymptotically tight.

Proof. Let $d$ be the diameter of $G$. By the theorem above,

$$
D^{\prime}(G) \leq \frac{1}{4} d n\left(n-\frac{d}{3}(\delta+1)\right)^{2}+O\left(n^{3}\right)
$$


The term $\frac{1}{4} d n(n-(d / 3)(\delta+1))^{2}$ is maximised, with respect to $d$, for $d=n /(\delta+1)$, to give

$$
\frac{1}{4} d n\left(n-\frac{d}{3}(\delta+1)\right)^{2} \leq \frac{n^{4}}{9(\delta+1)} .
$$

Hence $D^{\prime}(G) \leq n^{4} /(9(\delta+1))+O\left(n^{3}\right)$, as desired.

To see that the bound is asymptotically best possible, consider the graph $G_{n, d, \delta}$ constructed above. Note that

$$
D^{\prime}\left(G_{n, n /(\delta+1), \delta}\right)>\frac{n^{4}}{9(\delta+1)},
$$

as claimed.

\section{Open problems}

Since the extremal graph in Corollary 2.5 has cut vertices, it would be interesting to determine a best upper bound on the degree distance in terms of order and vertex connectivity. As reported in [4], the determination of the maximum degree distance among graphs of given order $n$ and size $m$ seems a difficult problem. At present the problem has only been solved for two special cases, unicyclic graphs (see Hou and Chang [7]) and bicyclic graphs (see Ilič et al. [8]). Lastly, it would be interesting to find a proof that is not so lengthy, elaborate, and delicate as the one presented in this paper.

\section{References}

[1] O. Bucicovschi and S. M. Cioabă, 'The minimum degree distance of graphs of given order and size', Discrete Appl. Math. 156 (2008), 3518-3521.

[2] P. Dankelmann and R. C. Entringer, 'Average distance, minimum degree and spanning trees', J. Graph Theory 33 (2000), 1-13.

[3] P. Dankelmann, I. Gutman, S. Mukwembi and H. C. Swart, 'On the degree distance of a graph', Discrete Appl. Math. 157 (2009), 2773-2777.

[4] P. Dankelmann and S. Mukwembi, 'The distance concept and distance in graphs', in: Distance in Molecular Graphs - Theory (eds. B. Furtula and I. Gutman) (University of Kragujevac, Kragujevac, 2012), pp. 2-48.

[5] A. A. Dobrynin and A. A. Kochetova, 'Degree distance of a graph: a degree analogue of the Wiener index', J. Chem. Inf. Comput. Sci. 34 (1994), 1082-1086.

[6] I. Gutman, 'Selected properties of the Schultz molecular topological index', J. Chem. Inf. Comput. Sci. 34 (1994), 1087-1089.

[7] Y. Hou and A. Chang, 'The unicyclic graphs with maximum degree distance', J. Math. Study 39 (2006), 18-24.

[8] A. Ilić, D. Stevanović, L. Feng, G. Yu and P. Dankelmann, 'Degree distance of unicyclic and bicyclic graphs', Discrete Appl. Math. 159 (2011), 779-788.

[9] D. J. Klein, Z. Mihalić, D. Plavšić and N. Trinajstić, 'Molecular topological index, a relation with the Wiener index', J. Chem. Inf. Comput. Sci. 32 (1992), 304-305.

[10] M. Kouider and P. Winkler, 'Mean distance and minimum degree', J. Graph Theory 25 (1997), 95-99. 
[11] Z. Mihalić, S. Nikolić and N. Trinajstić, 'Comparative study of molecular descriptors derived from the distance matrix', J. Chem. Inf. Comput. Sci. 32 (1992), 28-37.

[12] H. P. Schultz, E. B. Schultz and T. P Schultz, 'Topological organic chemistry 7. Graph theory and molecular topological indices of unsaturated and aromatic hydrocarbons', J. Chem. Inf. Comput. Sci. 33 (1993), 863-867.

[13] I. Tomescu, 'Some extremal properties of the degree distance of a graph', Discrete Appl. Math. 98 (1999), 159-163.

[14] A. I. Tomescu, 'Unicyclic and bicyclic graphs having minimum degree distance', Discrete Appl. Math. 156 (2008), 125-130.

[15] H. Wiener, 'Structural determination of the paraffin boiling points', J. Amer. Chem. Soc. 69 (1947), $17-20$.

S. MUKWEMBI, School of Mathematics, Statistics and Computer Science, University of KwaZulu-Natal, South Africa

e-mail: mukwembi@ukzn.ac.za

S. MUNYIRA, Department of Mathematics, University of Zimbabwe, Zimbabwe 